Prepared in cooperation with the

UTAH DEPARTMENT OF NATURAL RESOURCES, DIVISION OF OIL, GAS, AND MINING

\title{
Methane Gas Concentration in Soils and Ground Water, Carbon and Emery Counties, Utah, 1995-2003
}

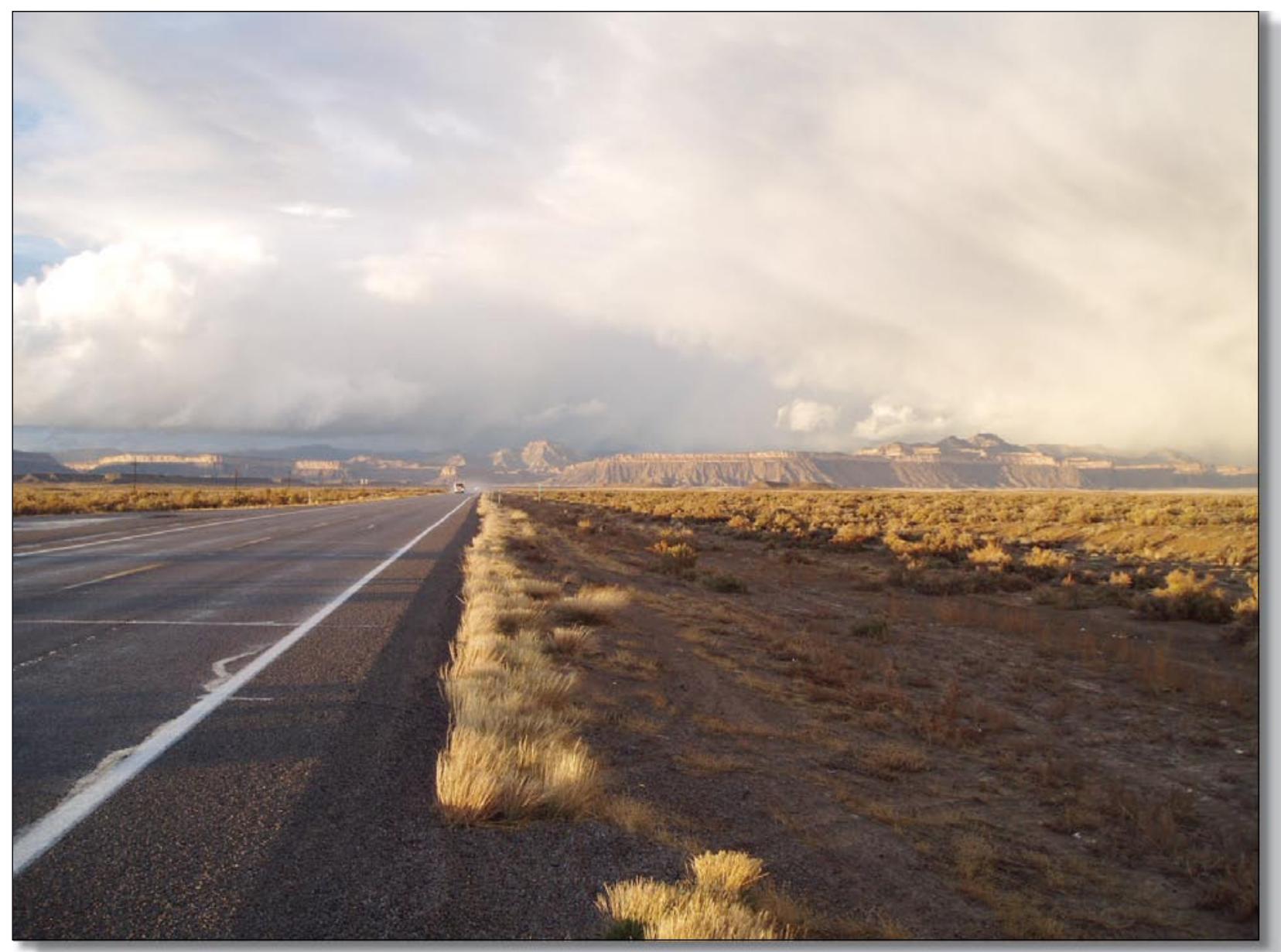

Scientific Investigations Report 2006-5227

U.S. Department of the Interior

U.S. Geological Survey 
Cover: Photograph of Book Cliffs in east-central Utah. Taken by B.J. Stolp. 


\title{
Methane Gas Concentration in Soils and Ground Water, Carbon and Emery Counties, Utah, 1995-2003
}

\author{
By B.J. Stolp, A.L. Burr, and K.K. Johnson
}

Scientific Investigations Report 2006-5227 


\title{
U.S. Department of the Interior \\ DIRK KEMPTHORNE, Secretary
}

\author{
U.S. Geological Survey \\ Mark D. Myers, Director
}

U.S. Geological Survey, Reston, Virginia: 2006

For product and ordering information:

World Wide Web: http://www.usgs.gov/pubprod

Telephone: 1-888-ASK-USGS

For more information on the USGS--the Federal source for science about the Earth, its natural and living resources, natural hazards, and the environment:

World Wide Web: http://www.usgs.gov

Telephone: 1-888-ASK-USGS

Any use of trade, product, or firm names is for descriptive purposes only and does not imply endorsement by the U.S. Government.

Although this report is in the public domain, permission must be secured from the individual copyright owners to reproduce any copyrighted materials contained within this report. 


\section{Contents}

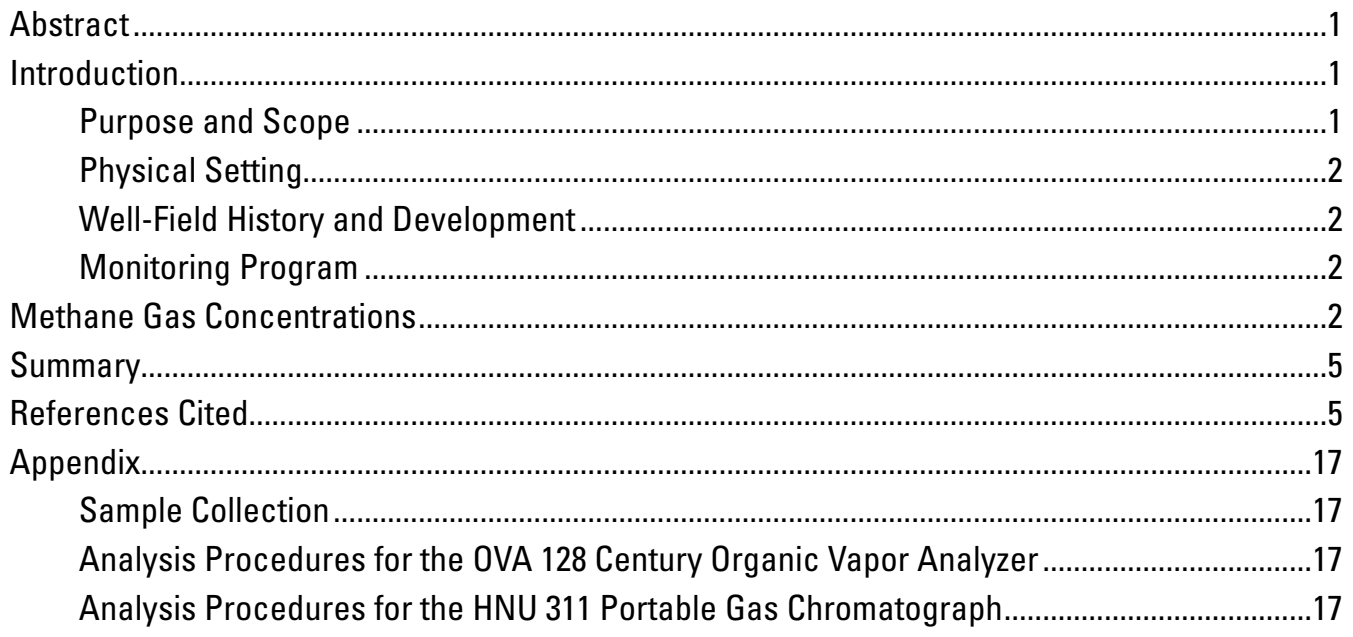

\section{Figures}

Figure 1. Generalized area of coal-bed methane development, Carbon and Emery Counties, Utah, 1985-2003.

Figure 2. Number of coal-bed methane gas-production wells and annual methane-gas production from the Ferron coal trend, Carbon and Emery Counties, Utah, 1985-2003

Figure 3. Location of sites where samples were collected and analyzed for methane concentration in Carbon and Emery Counties, Utah, 1995-2003

Figure 4. Number of methane-gas monitoring sites measured annually, Carbon and Emery Counties, Utah, 1995-2003.

Figure 5. Long-term trend at monitoring sites at which the highest concentrations of soil-gas methane were measured, Carbon and Emery Counties, Utah, 1995-2003

\section{Tables}

Table 1. Summary statistics of methane concentration for five areas of coal-bed production, Carbon and Emery Counties, Utah, 1995-2003

Table 2. Soil-gas methane concentration in samples collected adjacent to selected well sites, Carbon and Emery Counties, Utah, 1995-2003

Table 3. Soil-gas methane concentration in multiple samples collected adjacent to selected well sites, Carbon and Emery Counties, Utah, 1995-2003

Table 4. Soil-gas methane concentration in samples collected at selected groundwater sites, Carbon and Emery Counties, Utah, 1995-2002 
iv

\section{CONVERSION FACTORS, HORIZONTAL DATUM, AND ABBREVIATED WATER-OUALITY UNITS}

\begin{tabular}{|c|c|c|}
\hline Multiply & By & To obtain \\
\hline inch (in.) & 2.54 & centimeter $(\mathrm{cm})$ \\
\hline foot $(\mathrm{ft})$ & 0.3048 & meter $(\mathrm{m})$ \\
\hline
\end{tabular}

Horizontal coordinate information is referenced to the North American datum of 1983 (NAD 83).

Methane gas concentration is reported in parts per million by volume (ppmv). As an example, an air sample with a methane concentration of 1,000 ppmv would contain 1,000 molecules of methane for every $1,000,000$ molecules of air. 


\title{
Methane Gas Concentration in Soils and Ground Water, Carbon and Emery Counties, Utah, 1995-2003
}

\author{
By B.J. Stolp, A.L. Burr, and K.K. Johnson
}

\section{Abstract}

The release of methane gas from coal beds creates the potential for it to move into near-surface environments through natural and human-made pathways. To help ensure the safety of communities and determine the potential effects of development of coal-bed resources, methane gas concentrations in soils and ground water in Carbon and Emery Counties, Utah, were monitored from 1995 to 2003. A total of 420 samples were collected, which contained an average methane concentration of 2,740 parts per million by volume (ppmv) and a median concentration of less than 10 ppmv. On the basis of spatial and temporal methane concentration data collected during the monitoring period, there does not appear to be an obvious, widespread, or consistent migration of methane gas to the near-surface environment.

\section{Introduction}

The release of methane gas from coal beds creates the potential for it to move into near-surface environments through natural and human-made pathways. Natural pathways include fractures through the rock layers and voids between the grains of rock. Human-made pathways can be created when different types of wells are drilled. Methane is a combustable gas and can catch on fire if airborne concentrations exceed 5 percent (Eltschlager and others, 2001, p. 34-37). To help ensure the safety of communities and determine the potential effects of development of coal-bed resources, the U.S. Geological Survey and the Utah Department of Natural Resources, Division of Oil, Gas, and Mining, cooperated in a program to monitor methane gas concentration in soils and ground water in areas of coal-bed methane production. Initial findings from the monitoring program are discussed in a fact sheet by Naftz and others (1998).

Monitoring of methane gas in soils and ground water at selected locations in Carbon and Emery counties in Utah began in 1995 in response to commercial development of coal-bed methane resources from geologic formations that extend along the western edge of the San Rafael Swell in an area commonly referred to as the Ferron coal trend. The coal trend extends underneath the towns of Price, Huntington, Castle Dale, Ferron, and Emery (fig. 1). Methane is generated as a byproduct during formation of coal and is released as gas when ground water is pumped from geologic formations that contain coal beds. Boreholes are used to remove ground water and recover liberated methane. Substantial amounts of methane have been recovered from the Ferron trend coal beds since the early 1990 s.

\section{Purpose and Scope}

This report tabulates, summarizes, and interprets the results of methane monitoring of soils and ground water in Carbon and Emery Counties, Utah, from 1995 to 2003. A description of collection and analytical procedures used for soil gas and ground water samples is presented in the appendix.

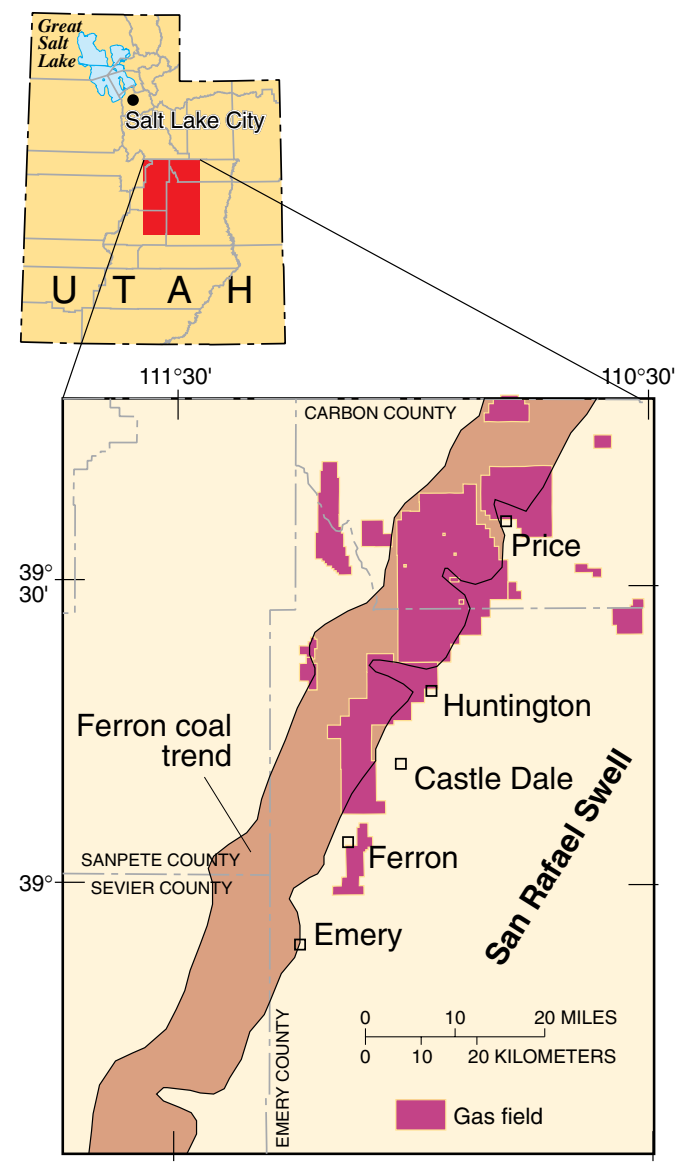

Figure 1. Generalized area of coal-bed methane development, Carbon and Emery Counties, Utah, 1985-2003. 


\section{Physical Setting}

Coal originates as plant matter deposited in swamp-like environments that then decays as it is buried and compressed over geologic time. Methane from the Ferron coal trend is produced from coal beds and sandstone in the Ferron Sandstone Member of the Mancos Shale and in the Blackhawk Formation of the Upper Cretaceous Mesaverde Group. Depth to these formations from land surface ranges from 1,000 to $4,500 \mathrm{ft}$ (Tabet and others, 1995; Stevens, 1993). Methane is recovered through boreholes that are drilled into the formations with a variety of different drilling, completion, and extraction technologies (Stevens, 1993). Gas is released when hydrostatic pressure created by overlying water is reduced. Hydrostatic pressure is reduced by pumping water out of the formations. Almost all of this water is currently (2006) disposed of by injecting it into geologic formations located thousands of feet below the Measverde Group.

\section{Well-Field History and Development}

Pilot development of coal-bed methane resources began near Price, Utah, in 1985. By 1991, methane production had spread south toward Orangeville and Ferron and as of November 2003, there were 772 coal-bed methane wells. Regulatory agencies continue (2006) to receive applications to drill additional coal-bed methane wells in the area, although at a much slower pace than in the past. The eventual number of wells will be determined primarily by the physical and economic limitations of the gas resource.

Peak production occurred in 2002 and was about 102 billion cubic feet of methane for the year. Gas production from 1985 through 2003 is shown in decatherms in figure 2. Decatherm is the unit used in most natural gas billing and is a measure of the heat produced when methane is ignited. The conversion from volume of methane gas to decatherm is made with the assumption that the heat value of methane from the Ferron coal trend is 1,000 British Thermal Units (BTU) per cubic foot of methane.

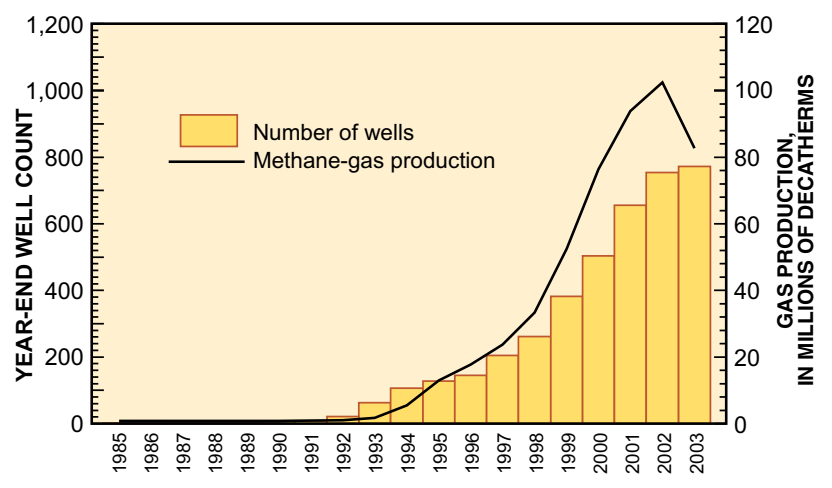

Figure 2. Number of coal-bed methane gas-production wells and annual methane-gas production from the Ferron coal trend, Carbon and Emery Counties, Utah, 1985-2003.

\section{Monitoring Program}

The monitoring described in this report began in 1995 and lasted through 2003. The program focused on established production-well sites and methane-gas production fields near residential areas. A priority was to identify and quantify trends and establish baseline methane concentrations in the soil and (or) ground water for future comparison. A core of twenty perimeter sites was established around Price, Huntington, Orangeville, and Ferron (fig. 3). These sites were monitored annually to determine whether or not methane concentrations were increasing near populated areas. Additional sites were established to monitor expanding areas of production and newly drilled production wells. A total of 420 shallow (2- to 4-ft depth) soil-gas and ground-water samples were collected from 174 soil and 15 ground-water sites (figs. 3 and 4). Summary statistics of methane concentrations for five areas of coal-bed production (fig. 3) are listed in table 1. Multiple samples were collected at 75 sites. Data describing site locations, sampling specifics, and methane concentrations are reported in tables 2-4, located at the back of this report. Sample collection and analysis procedures are described in the appendix, which is also located at the back of this report.

\section{Methane Gas Concentrations}

The average measured methane concentration for the monitoring period (1995-2003) was 2,740 parts per million by volume (ppmv); the median concentration was less than 10 ppmv. For calculation of summary statistics, sample values below the minimum detection limit or greater than the maximum detection limit were assigned the value of the detection limit. Two hundred and ten samples (52 percent of the sample set) had concentrations that were less than the detection limit. Twenty samples (5 percent of the sample set) had concentrations greater than 10,000 ppmv. On the basis of spatial and temporal methane concentration data collected during the monitoring period, there does not appear to be an obvious, widespread, or consistent migration of methane gas to the near-surface environment.

Inside buildings, methane-gas levels greater than 10,000 ppmv are potentially dangerous; in open air, methane levels above 50,000 ppmv can result in combustion of the air (Eltschlager and others, 2001, p. 34-37). At a concentration of less than 5,000 ppmv, methane poses no immediate threat (Eltschlager and others, 2001, p. 34-37).

Summary statistics of methane concentrations for five areas of coal-bed production (fig. 3) are listed in table 1. None of the production areas had average concentrations that are considered to be dangerous (greater than 5,000 ppmv). The median concentration for all areas is less than the average value, indicating that most values in the sample set are less than that average value. Twenty samples from 11 sites had methane concentrations that exceeded 10,000 ppmv. Those 


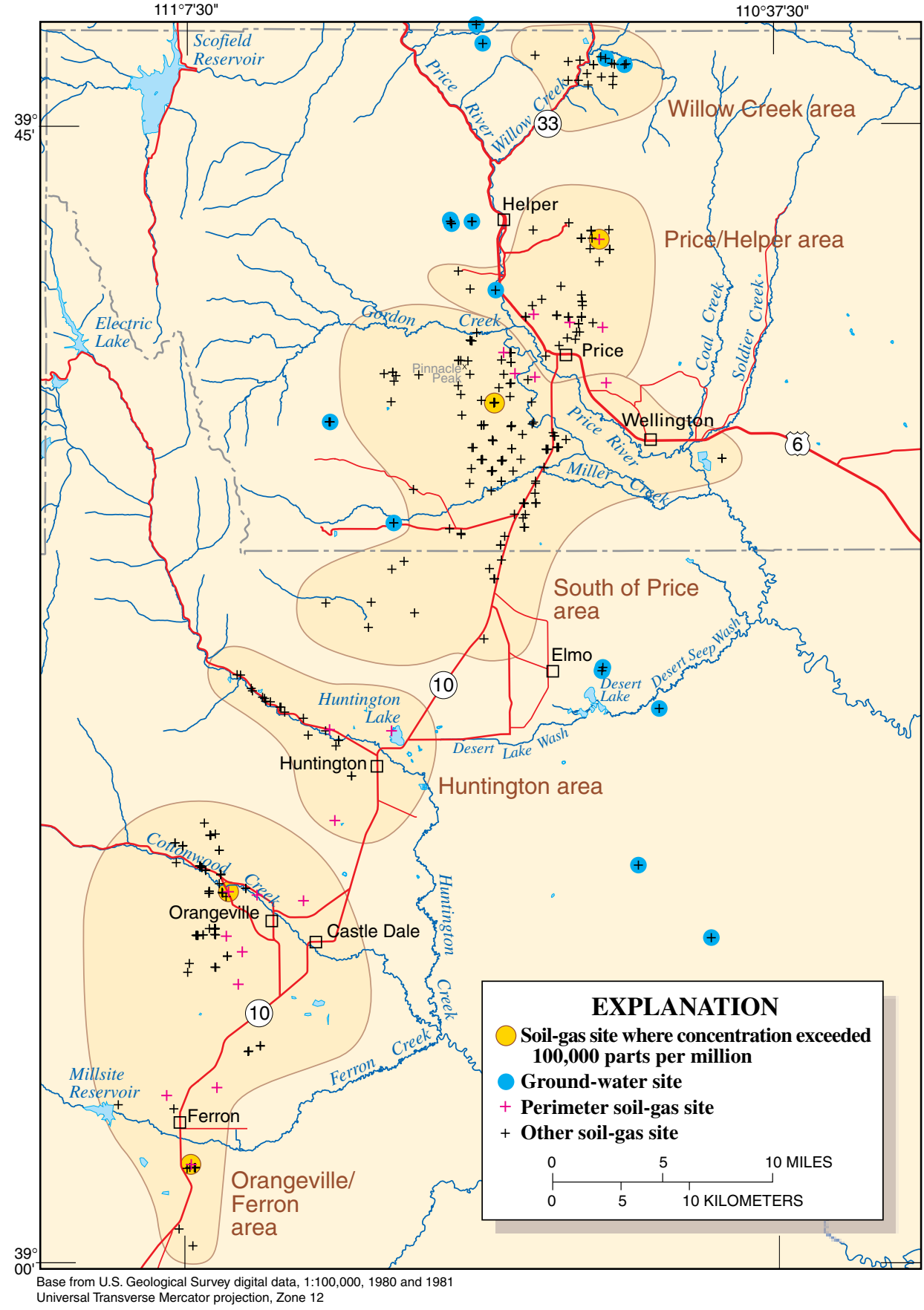

Figure 3. Location of sites where samples were collected and analyzed for methane concentration in Carbon and Emery Counties, Utah, 1995-2003. 


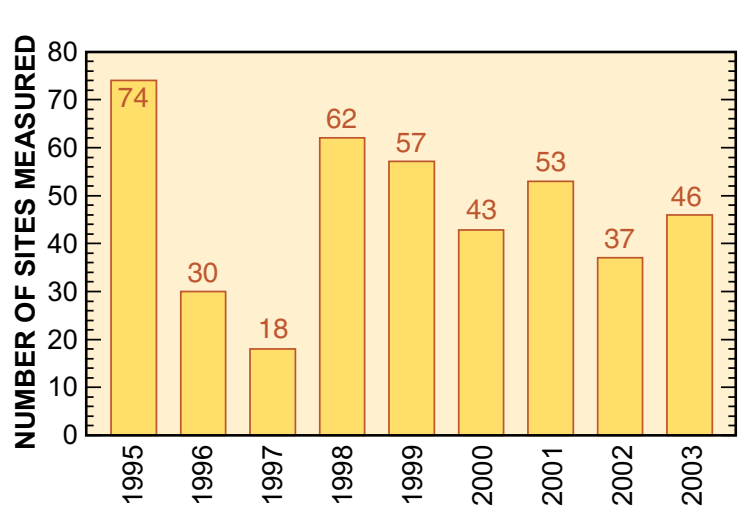

Figure 4. Number of methane-gas monitoring sites measured annually, Carbon and Emery Counties, Utah, 1995-2003.

sites are scattered across the entire area and do not indicate specific locations of high concentrations.

At 75 sites, soil-gas samples were collected multiple times during the monitoring period. These data show a general trend of decreasing methane concentration over time. At sites where a concentration of 10,000 ppmv or greater was measured at some time during the monitoring period, the most recent repeat measurements averaged 23 ppmv methane. Methane concentrations over time at sites with the highest concentrations (greater than 100,000 ppmv) are shown in figure 5 . These high concentrations might be associated with disturbances to the geohydrologic conditions that occur during drilling of coal-bed gas wells or problems with well maintenance. High methane concentrations are commonly observed shortly after drilling. If wells are properly constructed and maintained, concentrations that result from drilling geneally decrease and remain low over time.

At 15 of the 75 sites where temporal data were collected, soil-gas methane concentrations were inconsistent. Variations on the order of 10 to $1,000 \mathrm{ppmv}$ were measured from year to year and showed no consistent increasing or decreasing trends. Eight of these sites are located in the Orangeville/Ferron area. Changes of this type and magnitude, associated with this monitoring program, are likely a result of (1) intermittent

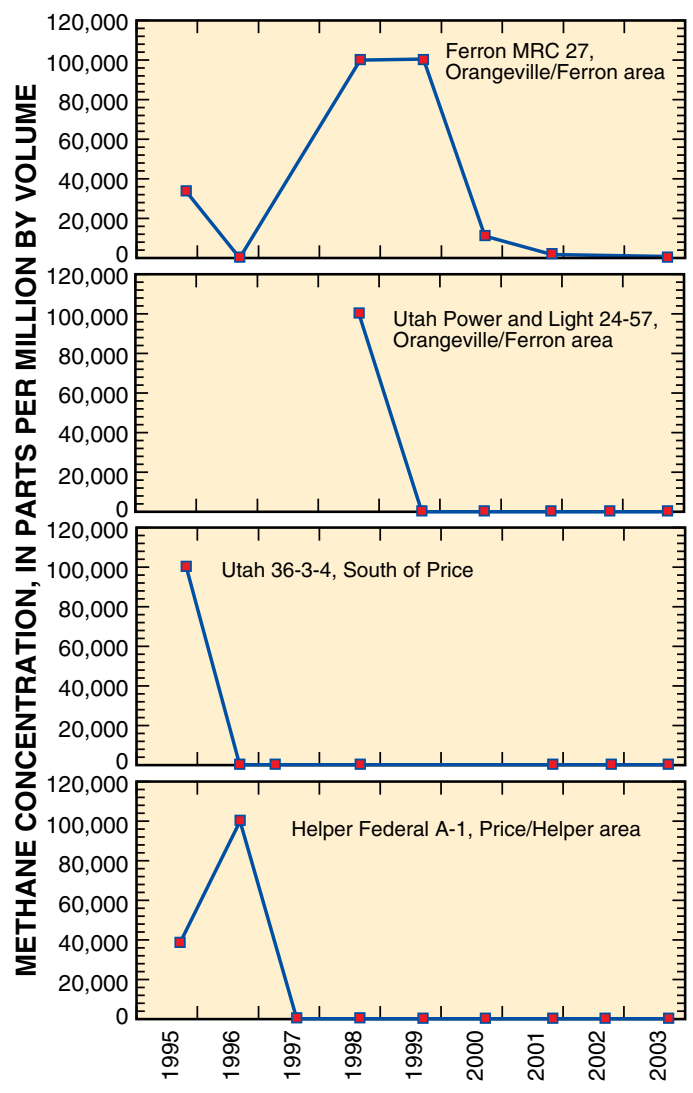

Figure 5. Long-term trend at monitoring sites at which the highest concentrations of soil-gas methane were measured, Carbon and Emery Counties, Utah, 1995-2003.

pumping at the methane-gas well prior to sampling; (2) variations in climatic conditions including temperature, barometric pressure, and soil moisture (Rose and others, 1979); and (3) inherent uncertainties associated with field collection and analysis of soil-gas samples (table 3).

Monitoring of methane concentration in ground water was limited. An initial inventory of coal-bed methane production areas in 1995 resulted in sample collection from 14 sites that included springs, drains, and ponds. The average methane concentration was 20 ppmv. Multiple samples were collected at a single spring from 2000 through 2002. Methane concen-

Table 1. Summary statistics of methane concentration for five areas of coal-bed production, Carbon and Emery Counties, Utah, 1995-2003

[ppmv, parts per million by volume; <, concentration is less than the minimum detection limit of the analytical equipment; >, concentration is greater than the minimum detection limit of the analytical equipment]

\begin{tabular}{lcccrr}
\hline \multicolumn{1}{c}{ Area } & $\begin{array}{c}\text { Average } \\
\text { concentration } \\
\text { (ppmv) }\end{array}$ & $\begin{array}{c}\text { Median } \\
\text { concentration } \\
\text { (ppmv) }\end{array}$ & $\begin{array}{c}\text { Minimum } \\
\text { concentration } \\
\text { (ppmv) }\end{array}$ & $\begin{array}{c}\text { Maximum } \\
\text { concentration } \\
\text { (ppmv) }\end{array}$ & $\begin{array}{c}\text { Number } \\
\text { of } \\
\text { samples }\end{array}$ \\
\hline Willow Creek & 800 & $<10$ & $<10$ & 6,390 & 15 \\
Price/Helper & 2,260 & 10 & $<1$ & $>100,000$ & 75 \\
South of Price & 1,750 & $<10$ & $<1$ & $>100,000$ & 160 \\
Huntington & 15 & 4 & $<1$ & $<1$ & 24 \\
Orangeville/Ferron & 4,920 & $<10$ & $<100,000$ & 137 \\
\hline
\end{tabular}


tration at that site decreased from 26,200 ppmv in 2000 to 160 ppmv in 2002.

\section{Summary}

To help ensure the safety of communities and determine the potential effects of development of coal-bed resources, the U.S. Geological Survey and the Utah Department of Natural Resources, Division of Oil, Gas, and Mining, cooperated in a program to monitor methane concentrations in soils and ground water in areas of coal-bed methane production. The monitoring program began in 1995 and lasted through 2003. Commercial development of coal-bed methane resources began in the early 1990s from geologic formations within the Ferron coal trend. The monitoring program focused on established production-well sites and methane-gas production fields near residential areas. A core of twenty perimeter sites was established around Price, Huntington, Orangeville, and Ferron and monitored annually. Additional sites were established to monitor expanding areas of production and newly drilled production wells. A total of 420 shallow (2- to 4-ft depth) soilgas and ground-water samples were collected from 174 soil and 15 ground-water sites. Multiple samples were collected at 75 sites. The average measured methane concentration for the monitoring period was 2,740 parts per million by volume (ppmv); the median concentration was less than 10 ppmv. On the basis of spatial and temporal methane concentration data collected during the monitoring period, there does not appear to be an obvious, widespread, or consistent migration of methane gas to the near-surface environment.

\section{References Cited}

Chafin, D.T., Swanson, D.H., and Grey, D.W., 1993, Methaneconcentration and methane-isotope data for ground water and soil gas in the Animas River Valley, Colorado and New Mexico, 1990-91: U.S. Geological Survey Water-Resources Investigations Report 93-4007, 86 p.

Eltschlager, K.K., Hawkins, J.W., Ehler, W.C., and Baldassare, Fred, 2001, Technical measures for the investigation and mitigation of fugitive methane hazards in areas of coal mining: Pittsburgh, Pa., U.S. Department of the Interior, Office of Surface Mining Reclamation and Enforcement, Appalachian Regional Coordinating Center, 124 p., accessed May 22, 2006, at http://www.osmre.gov/rep.htm

Naftz, D.L., Hadley, H.K., and Hunt, G.L., 1998, Determination of methane concentrations in shallow ground water and soil gas near Price, Utah: U.S. Geological Survey Fact Sheet FS-191-97, 4 p.

Rose, A.W., Hawkes, H.E., and Webb, J.S., 1979, Geochemistry in mineral exploration ( $2^{\text {nd }}$ ed.): New York, Academic Press, $657 \mathrm{p}$.

Stevens, S.H., 1993, Exploration and development, Uinta Basin, Utah: Quarterly Review of Methane from Coal Seams Technology, v. 11, no. 1, p. 38-42.

Tabet, D.E., Hucka, B.P., Sommer, S.N., 1995, Maps of total Ferron coal, depth to top, and vitrinite reflectance for the Ferron Sandstone Member of the Mancos Shale, central Utah: Utah Geological Survey Open-File Report 329, 3 pls., scale 1:250,000. 
Table 2. Soil-gas methane concentration in samples collected adjacent to selected well sites, Carbon and Emery Counties, Utah, 1995-2003

[Site name: Utah Division of Oil, Gas, and Mining well designations or field notes. Location: d, degree; m, minute; s, second. Methane concentration: ppmv, parts per million by volume. Samples collected from 1995 through 2000 were analyzed by the OVA 128 Century Organic Vapor Analyzer, which was considered for this monitoring program to have a minimum detection limit of 10 ppmv. Samples collected from 2001 through 2003 were analyzed by the HNU Systems Inc. Model 311 Portable Gas Chromatograph, which was considered for this monitoring program to have a minimum detection limit of 1 ppmv. When no methane was detected at a sample site, a value of either less than 10 ppmv $(<10)$ or less than 1 ppmv $(<1)$ is reported. A dash $(-)$ indicates no sample was collected. Area: Refers to areas delineated in figure 3]

\begin{tabular}{|c|c|c|c|c|c|c|}
\hline \multirow[b]{2}{*}{ Site name } & \multicolumn{2}{|c|}{ Location } & \multirow[b]{2}{*}{$\begin{array}{l}\text { Sample } \\
\text { date }\end{array}$} & \multicolumn{2}{|c|}{$\begin{array}{l}\text { Methane concentration } \\
\text { (ppmv) }\end{array}$} & \multirow[b]{2}{*}{ Area } \\
\hline & $\begin{array}{l}\text { Latitude } \\
\text { (ddmmss) }\end{array}$ & $\begin{array}{l}\text { Longitude } \\
\text { (dddmmss) }\end{array}$ & & $\begin{array}{c}\text { Sample } \\
\text { collected } \\
\text { within } 10 \text { feet } \\
\text { of well } \\
\end{array}$ & $\begin{array}{c}\text { Sample } \\
\text { collected more } \\
\text { than } 10 \text { feet } \\
\text { from well }\end{array}$ & \\
\hline Helper Federal A-3 & 394040 & 1104652 & 09/06/1995 & $<10$ & - & Price/Helper \\
\hline Washington Park & 393625 & 1104834 & 09/07/1995 & $<10$ & - & Price/Helper \\
\hline City Park, Price & 393640 & 1104744 & 09/07/1995 & $<10$ & - & Price/Helper \\
\hline River Gas Well 30-5-15 & 393455 & 1105105 & 09/08/1995 & $<10$ & - & South of Price \\
\hline River Gas Well 24-16-18 & 393517 & 1105124 & 09/08/1995 & $<10$ & - & South of Price \\
\hline River Gas Well 23-4-20 & 393549 & 1105314 & 09/08/1995 & $<10$ & - & South of Price \\
\hline River Gas Well 24-2-15 & 393551 & 1105131 & 09/08/1995 & $<10$ & - & South of Price \\
\hline River Gas Well 35-1-9 & 393415 & 1105233 & 09/12/1995 & $<10$ & - & South of Price \\
\hline River Gas Well 27-9-30 & 393429 & 1105336 & 09/12/1995 & $<10$ & - & South of Price \\
\hline River Gas Well 27-8-29 & 393450 & 1105344 & 09/12/1995 & $<10$ & - & South of Price \\
\hline River Gas Well 23-14-21 & 393517 & 1105308 & 09/12/1995 & $<10$ & - & South of Price \\
\hline River Gas Well 24-80 & 393040 & 1105203 & 09/13/1995 & $<10$ & - & South of Price \\
\hline River Gas Well 14-64 & 393058 & 1105309 & 09/13/1995 & $<10$ & - & South of Price \\
\hline River Gas Well 6-40 & 393254 & 1105027 & 09/13/1995 & $<10$ & - & South of Price \\
\hline River Gas Well 18-69 & 393127 & 1105056 & 09/14/1995 & $<10$ & - & South of Price \\
\hline River Gas Well 7-59 & 393155 & 1105032 & 09/14/1995 & $<10$ & - & South of Price \\
\hline River Gas Well 30-14-14 & 393424 & 1105105 & 09/14/1995 & $<10$ & - & South of Price \\
\hline Federal 16-15 & 394644 & 1104703 & 09/18/1995 & $<10$ & - & Willow Creek \\
\hline Shimmin Trust 3 & 394703 & 1104622 & 09/18/1995 & $<10$ & - & Willow Creek \\
\hline Jensen $7-15$ & 394711 & 1104706 & 09/18/1995 & 120 & - & Willow Creek \\
\hline Shimmin Trust $11-11$ & 394751 & 1104624 & 09/18/1995 & $<10$ & - & Willow Creek \\
\hline $\begin{array}{l}\text { Jensen 11-15 near Highway } 191 \\
\text { (between mile } 163 \text { and164) }\end{array}$ & 394655 & 1104733 & 09/19/1995 & $<10$ & - & Willow Creek \\
\hline Utah 9-16 & 394655 & 1104804 & 09/19/1995 & $<10$ & - & Willow Creek \\
\hline Shimmin Trust 14-12 & 394734 & 1104506 & 09/19/1995 & $<10$ & - & Willow Creek \\
\hline Shimmin Trust 1 & 394736 & 1104540 & 09/19/1995 & 2,840 & - & Willow Creek \\
\hline Jensen $16-9$ & 394740 & 1104804 & 09/19/1995 & $<10$ & - & Willow Creek \\
\hline Jensen $11-10$ & 394743 & 1104728 & 09/19/1995 & 2,420 & - & Willow Creek \\
\hline Federal 6-8 & 394755 & 1104946 & 09/19/1995 & $<10$ & - & Willow Creek \\
\hline Utah 25-7-6 & 393445 & 1105139 & 09/20/1995 & $<10$ & - & South of Price \\
\hline Federal 16-14 & 394645 & 1104547 & 09/20/1995 & $<10$ & - & Willow Creek \\
\hline Shimmin Trust 2 & 394704 & 1104546 & 09/20/1995 & 6,390 & - & Willow Creek \\
\hline Jenson $16-10$ & 394732 & 1104646 & 09/20/1995 & $<10$ & - & Willow Creek \\
\hline Abandoned Gas Well 1 & 392910 & 1105411 & 09/21/1995 & $<10$ & - & South of Price \\
\hline Abandoned Gas Well 2 & 390659 & 1110557 & $10 / 24 / 1995$ & 160 & - & Orangeville/Ferron \\
\hline Abandoned well marker & 392711 & 1105153 & $10 / 24 / 1995$ & $<10$ & - & South of Price \\
\hline MRC 21 & 390046 & 1110715 & $10 / 25 / 1995$ & $<10$ & - & Orangeville/Ferron \\
\hline Active gas well - Ferron & 390350 & 1110733 & $10 / 25 / 1995$ & $<10$ & - & Orangeville/Ferron \\
\hline Backyard 650 N 300 E & 393643 & 1104809 & 09/09/1996 & $<10$ & - & Price/Helper \\
\hline Helper Federal B-1 & 393846 & 1104828 & 09/09/1996 & $<10$ & - & Price/Helper \\
\hline Ferron MRC 17 & 390126 & 1110757 & 09/10/1996 & $<10$ & - & Orangeville/Ferron \\
\hline
\end{tabular}


Table 2. Soil-gas methane concentration in samples collected adjacent to selected well sites, Carbon and Emery Counties, Utah, 1995-2003-Continued

\begin{tabular}{|c|c|c|c|c|c|c|}
\hline \multirow[b]{2}{*}{ Site name } & \multicolumn{2}{|c|}{ Location } & \multirow[b]{2}{*}{$\begin{array}{l}\text { Sample } \\
\text { date }\end{array}$} & \multicolumn{2}{|c|}{$\begin{array}{l}\text { Methane concentration } \\
\text { (ppmv) }\end{array}$} & \multirow[b]{2}{*}{ Area } \\
\hline & $\begin{array}{l}\text { Latitude } \\
\text { (ddmmss) }\end{array}$ & $\begin{array}{l}\text { Longitude } \\
\text { (dddmmss) }\end{array}$ & & $\begin{array}{c}\text { Sample } \\
\text { collected } \\
\text { within } 10 \text { feet } \\
\text { of well }\end{array}$ & $\begin{array}{c}\text { Sample } \\
\text { collected more } \\
\text { than } 10 \text { feet } \\
\text { from well }\end{array}$ & \\
\hline Chandler Buzzard Bench Federal 3-24 & 391136 & 1110732 & $09 / 10 / 1996$ & $<10$ & - & Orangeville/Ferron \\
\hline Buzzard Bench Chandler Federal 6-3 & 391157 & 1110727 & 09/10/1996 & $<10$ & - & Orangeville/Ferron \\
\hline Utah Federal A 26-2 & 391320 & 1110626 & 09/10/1996 & $<10$ & - & Orangeville/Ferron \\
\hline SWD-1 Injection Well & 391436 & 1110535 & 09/10/1996 & $<10$ & - & Orangeville/Ferron \\
\hline Utah Federal K 5-34 & 392219 & 1110320 & $09 / 12 / 1996$ & $<10$ & - & Huntington \\
\hline River Gas $14-62$ & 393133 & 1105304 & $09 / 12 / 1996$ & $<10$ & - & South of Price \\
\hline Arcadia Telonis (D-2) Injection Well & 393514 & 1105655 & $09 / 12 / 1996$ & $<10$ & - & South of Price \\
\hline E.G. Telonis Etal \#1 & 393529 & 1105549 & $09 / 12 / 1996$ & $<10$ & - & South of Price \\
\hline Possibly Old CVA-12 site & 393258 & 1104853 & 09/13/1996 & $<10$ & - & South of Price \\
\hline Utah $19-222$ & 391448 & 110528 & 09/03/1998 & 50 & $<10$ & Orangeville/Ferron \\
\hline Helper Federal B-5 & 394105 & 1104553 & 04/08/1997 & $<10$ & - & Price/Helper \\
\hline Utah Federal H 6-21 & 390621 & 1111103 & 04/09/1997 & $<10$ & - & Orangeville/Ferron \\
\hline Utah Federal M 6-25 & 392230 & 1110344 & 04/09/1997 & $<10$ & - & Huntington \\
\hline River Gas 30-78 NE4/NE4 & 393504 & 1105010 & 04/09/1997 & $<10$ & - & South of Price \\
\hline Helper State SWD-1 & 393800 & 1104722 & 08/31/1998 & $<10$ & - & Price/Helper \\
\hline Helper State A-5 & 393810 & 1104723 & $08 / 31 / 1998$ & $<10$ & $<10$ & Price/Helper \\
\hline Helper State A-1 & 393825 & 1104725 & $08 / 31 / 1998$ & $<10$ & - & Price/Helper \\
\hline Birch A-1 & 393800 & 1104956 & 09/01/1998 & $<10$ & - & Price/Helper \\
\hline Federal 35-5 & 391306 & 1110610 & $09 / 02 / 1998$ & $<10$ & - & Orangeville/Ferron \\
\hline Utah 19-222 & 391448 & 1110528 & 09/03/1998 & 50 & $<10$ & Orangeville/Ferron \\
\hline Utah 36-135 & 392852 & 1105122 & 09/14/1999 & $<10$ & - & South of Price \\
\hline Robertson 32-127 & 393411 & 1104959 & 09/15/1999 & $<10$ & - & South of Price \\
\hline Kakatsides 31-197 & 393412 & 1105710 & 09/15/1999 & $<10$ & - & South of Price \\
\hline Telonis $30-156$ & 393501 & 1105701 & 09/15/1999 & $<10$ & - & South of Price \\
\hline Prettyman 11-114 & 393656 & 1105247 & 09/15/1999 & $<10$ & - & South of Price \\
\hline Woolstenhulme 5-266 & 393815 & 1104927 & 09/15/1999 & $<10$ & - & Price/Helper \\
\hline USA 30-289 & 392944 & 1105019 & 09/16/1999 & $<10$ & - & South of Price \\
\hline USA $20-287$ & 393033 & 1104952 & 09/16/1999 & $<10$ & - & South of Price \\
\hline Helper State A-13 & 393719 & 1104650 & 09/17/1999 & $<10$ & - & Price/Helper \\
\hline USA 2 & 392936 & 1105028 & $09 / 18 / 2000$ & $<10$ & - & South of Price \\
\hline Powell 30-173 & 392944 & 1105055 & $09 / 18 / 2000$ & $<10$ & - & South of Price \\
\hline Ferron 1-22 & 390352 & 1110727 & $09 / 19 / 2000$ & $<10$ & - & Orangeville/Ferron \\
\hline Nelson 1 & 390611 & 1110816 & $09 / 19 / 2000$ & $<10$ & - & Orangeville/Ferron \\
\hline Scorpion 1-23 & 392448 & 1105229 & $09 / 19 / 2000$ & $<10$ & - & South of Price \\
\hline USA 01-312 & 392756 & 1105135 & $09 / 19 / 2000$ & $<10$ & - & South of Price \\
\hline Utah 17-101 & 393135 & 1104924 & $09 / 19 / 2000$ & $<10$ & - & South of Price \\
\hline Utah Power and Light 24-51 & 391444 & 1110548 & $09 / 21 / 2000$ & $<10$ & - & Orangeville/Ferron \\
\hline Utah Power and Light 24-53 & 391507 & 1110559 & $09 / 21 / 2000$ & $<10$ & - & Orangeville/Ferron \\
\hline Utah U 2-49 & 391705 & 1110611 & $09 / 21 / 2000$ & $<10$ & - & Orangeville/Ferron \\
\hline Utah U 2-11 & 391731 & 1110644 & $09 / 21 / 2000$ & $<10$ & - & Orangeville/Ferron \\
\hline Gardner Trust 16-121 & 392100 & 1110124 & $10 / 26 / 2001$ & $<1$ & - & Huntington \\
\hline Utah BB 08-122 & 392206 & 1110251 & $10 / 26 / 2001$ & 8 & - & Huntington \\
\hline Huber-Shimmin Trust 2-11 & 394735 & 1104550 & $10 / 26 / 2001$ & 99 & - & Willow Creek \\
\hline Utah $17-370$ & 392550 & 1105558 & $10 / 30 / 2001$ & 4 & - & South of Price \\
\hline Utah 5-343 & 392753 & 1105631 & $10 / 30 / 2001$ & 2 & - & South of Price \\
\hline
\end{tabular}


Table 2. Soil-gas methane concentration in samples collected adjacent to selected well sites, Carbon and Emery Counties, Utah, 1995-2003-Continued

\begin{tabular}{|c|c|c|c|c|c|c|}
\hline \multirow[b]{2}{*}{ Site name } & \multicolumn{2}{|c|}{ Location } & \multirow[b]{2}{*}{$\begin{array}{l}\text { Sample } \\
\text { date }\end{array}$} & \multicolumn{2}{|c|}{$\begin{array}{l}\text { Methane concentration } \\
\text { (ppmv) }\end{array}$} & \multirow[b]{2}{*}{ Area } \\
\hline & $\begin{array}{l}\text { Latitude } \\
\text { (ddmmss) }\end{array}$ & $\begin{array}{l}\text { Longitude } \\
\text { (dddmmss) }\end{array}$ & & $\begin{array}{c}\text { Sample } \\
\text { collected } \\
\text { within } 10 \text { feet } \\
\text { of well }\end{array}$ & $\begin{array}{c}\text { Sample } \\
\text { collected more } \\
\text { than } 10 \text { feet } \\
\text { from well }\end{array}$ & \\
\hline William I 09-118 & 392140 & 1110142 & $09 / 11 / 2002$ & $<1$ & - & Huntington \\
\hline Conover 14-171 & 392034 & 1110002 & $09 / 23 / 2003$ & - & 2 & Huntington \\
\hline Utah Power and Light 06-104 & 392227 & 1110340 & $09 / 23 / 2003$ & - & 16 & Huntington \\
\hline Utah Power and Light 06-102 & 392250 & 1110421 & $09 / 23 / 2003$ & - & 9 & Huntington \\
\hline Utah T 36-100 & 392322 & 1110454 & $09 / 23 / 2003$ & - & 160 & Huntington \\
\hline Utah 1 & 393441 & 1105129 & $09 / 23 / 2003$ & - & $<1$ & South of Price \\
\hline Ferron Federal 4-36-18-7 & 391309 & 1110540 & $09 / 24 / 2003$ & - & 21 & Orangeville/Ferron \\
\hline Utah $24-560$ & 392516 & 1105822 & $09 / 24 / 2003$ & - & 4 & South of Price \\
\hline PPCO 15-555 & 392614 & 1110033 & $10 / 01 / 2003$ & - & 18 & South of Price \\
\hline Clawson Spring State H-2 & 392616 & 1105814 & $10 / 01 / 2003$ & - & 45 & South of Price \\
\hline Utah $20-333$ & 393043 & 1105604 & $10 / 01 / 2003$ & - & 43 & South of Price \\
\hline Utah 2-404 & 393840 & 1105309 & $10 / 01 / 2003$ & - & 40 & Price/Helper \\
\hline Utah $34-510$ & 393922 & 1105346 & $10 / 01 / 2003$ & - & 133 & Price/Helper \\
\hline Helper Federal E-6 & 394060 & 1104956 & $10 / 01 / 2003$ & - & 100 & Price/Helper \\
\hline Blackhawk A-4 & 394116 & 1104806 & $10 / 01 / 2003$ & - & 33 & Price/Helper \\
\hline
\end{tabular}


Table 3. Soil-gas methane concentration in multiple samples collected adjacent to selected well sites, Carbon and Emery Counties, Utah, 1995-2003

[Site name: Utah Division of Oil, Gas, and Mining well designations or field notes. Location: d, degree; m, minute; s, second. Methane concentration: ppmv, parts per million by volume. Samples collected from 1995 through 2000 were analyzed by the OVA 128 Century Organic Vapor Analyzer, which was considered for this monitoring program to have a minimum detection limit of 10 ppmv. Samples collected from 2001 through 2003 were analyzed by the HNU Systems Inc. Model 311 Portable Gas Chromatograph, which was considered for this monitoring program to have a minimum detection limit of 1 ppmv. When no methane was detected at a sample site, a value of either less than $10 \mathrm{ppmv}(<10)$ or less than 1 ppmv $(<1)$ is reported. Both analytical instruments were considered for this monitoring program to have a maximum detection limit of 100,000 ppmv. When analysis of a gas sample resulted in a value that exceeded 100,000 ppmv, the value is reported as greater than 100,000 (>100,000). A dash (-) indicates no sample was collected. Area: Refers to areas delineated in figure 3]

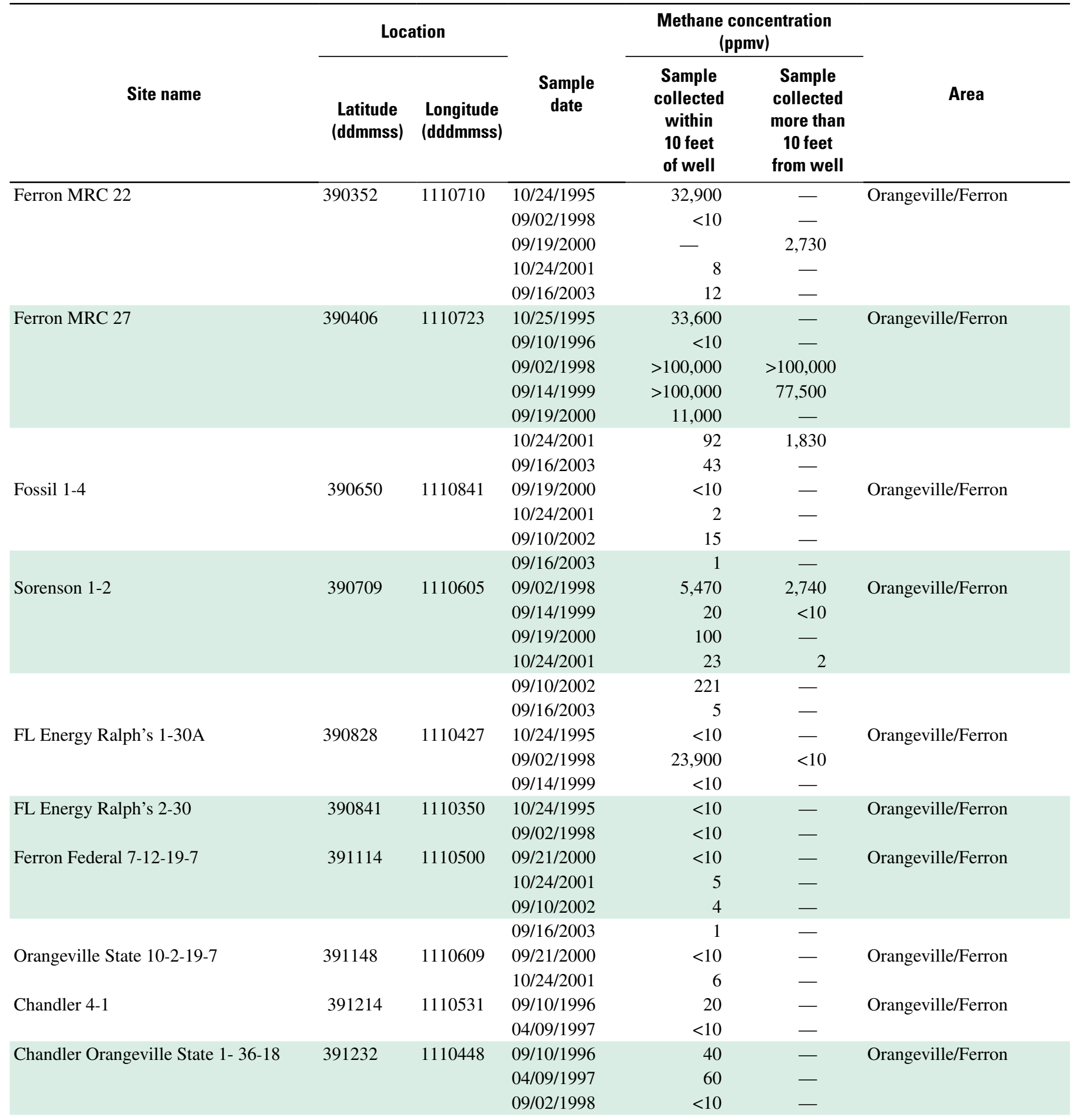


Table 3. Soil-gas methane concentration in multiple samples collected adjacent to selected well sites, Carbon and Emery Counties, Utah, 1995-2003-Continued

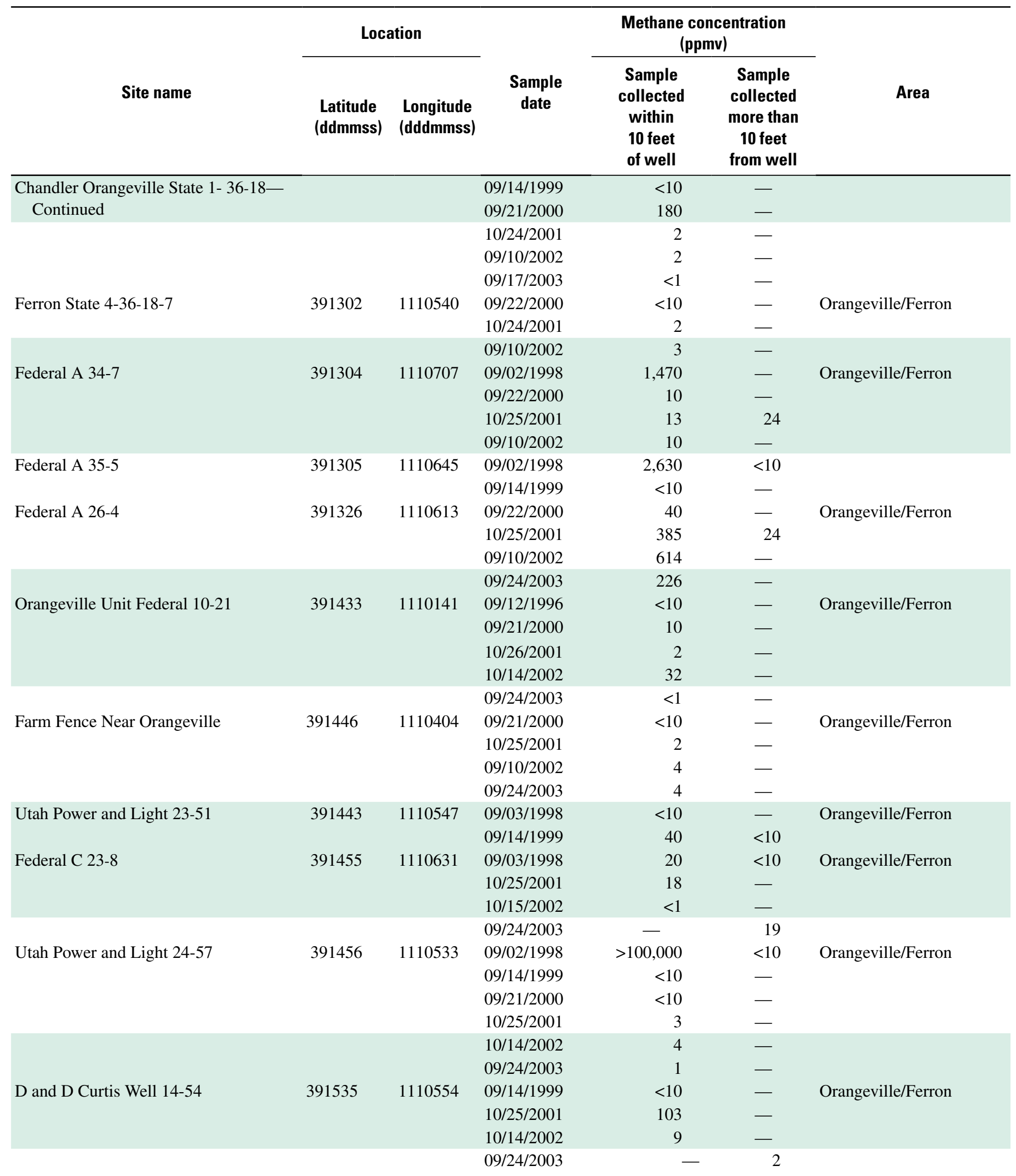


Table 3. Soil-gas methane concentration in multiple samples collected adjacent to selected well sites, Carbon and Emery Counties, Utah, 1995-2003-Continued

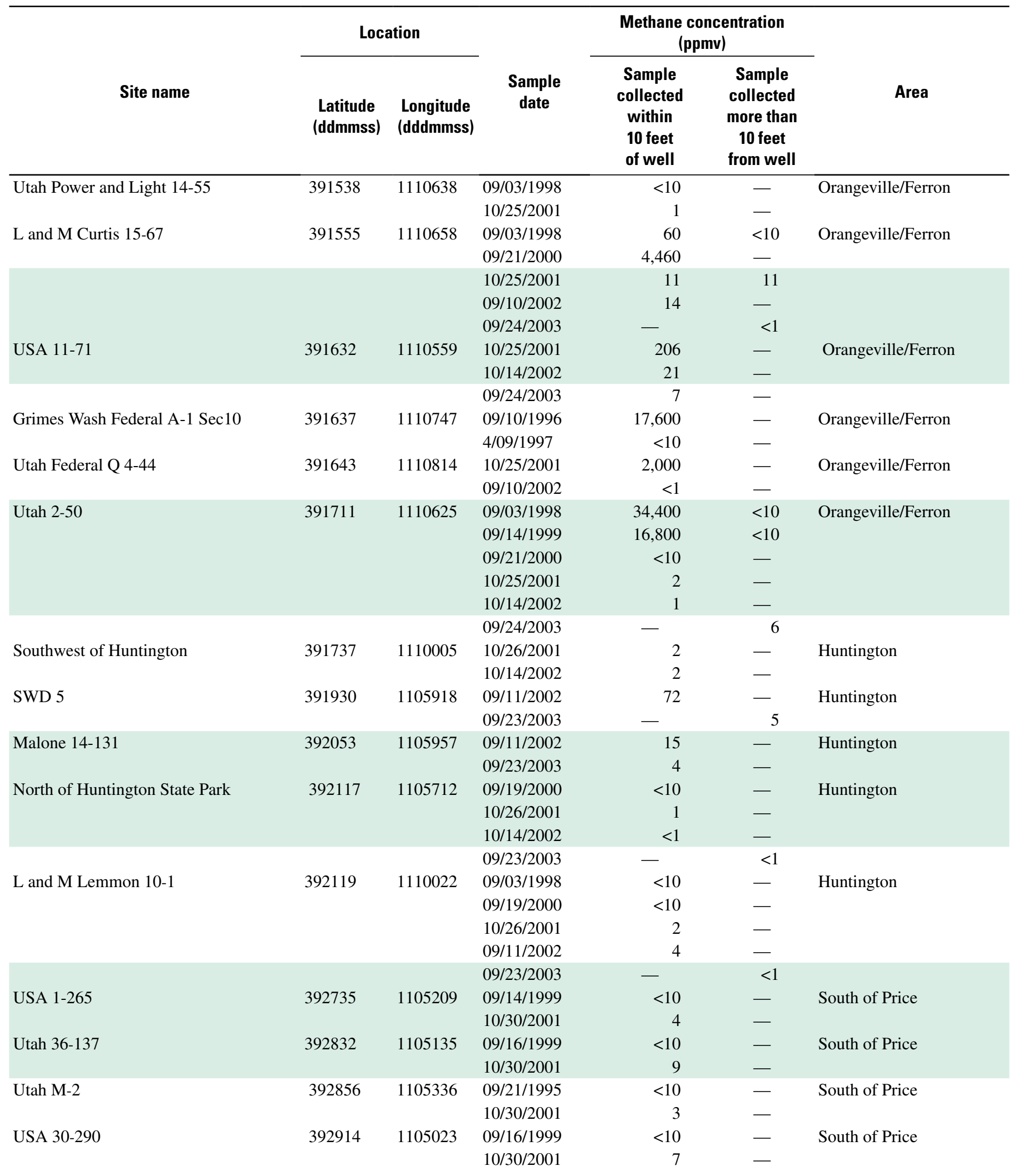


Table 3. Soil-gas methane concentration in multiple samples collected adjacent to selected well sites, Carbon and Emery Counties, Utah, 1995-2003-Continued

\begin{tabular}{|c|c|c|c|c|c|c|}
\hline \multirow[b]{2}{*}{ Site name } & \multicolumn{2}{|c|}{ Location } & \multirow[b]{2}{*}{$\begin{array}{l}\text { Sample } \\
\text { date }\end{array}$} & \multicolumn{2}{|c|}{$\begin{array}{c}\text { Methane concentration } \\
\text { (ppmv) }\end{array}$} & \multirow[b]{2}{*}{ Area } \\
\hline & $\begin{array}{l}\text { Latitude } \\
\text { (ddmmss) }\end{array}$ & $\begin{array}{l}\text { Longitude } \\
\text { (dddmmss) }\end{array}$ & & $\begin{array}{c}\text { Sample } \\
\text { collected } \\
\text { within } \\
10 \text { feet } \\
\text { of well }\end{array}$ & $\begin{array}{c}\text { Sample } \\
\text { collected } \\
\text { more than } \\
10 \text { feet } \\
\text { from well }\end{array}$ & \\
\hline \multirow[t]{2}{*}{ USA $20-288$} & 393011 & 1104949 & 09/16/1999 & 20 & $<10$ & South of Price \\
\hline & & & $09 / 18 / 2000$ & $<10$ & - & \\
\hline \multirow[t]{2}{*}{ USA $19-222$} & 393011 & 1105024 & 09/16/1999 & $<10$ & - & South of Price \\
\hline & & & $10 / 30 / 2001$ & 10 & - & \\
\hline \multirow[t]{2}{*}{ Utah 13-67 } & 393103 & 1105132 & 09/14/1995 & 20 & - & South of Price \\
\hline & & & 04/10/1997 & $<10$ & - & \\
\hline \multirow[t]{2}{*}{ Utah 17-103 } & 393058 & 1104947 & 09/11/1996 & $<10$ & - & South of Price \\
\hline & & & 09/16/1999 & $<10$ & - & \\
\hline \multirow[t]{6}{*}{ Utah 13-66 } & 393135 & 1105205 & 09/13/1995 & 4,850 & - & South of Price \\
\hline & & & $10 / 26 / 1995$ & $<10$ & - & \\
\hline & & & 09/11/1996 & $<10$ & - & \\
\hline & & & 04/10/1997 & $<10$ & - & \\
\hline & & & $10 / 16 / 2002$ & 3 & - & \\
\hline & & & $09 / 22 / 2003$ & 5 & - & \\
\hline \multirow[t]{4}{*}{ Utah 12-15-37 } & 393151 & 1105131 & 09/14/1995 & 260 & - & South of Price \\
\hline & & & 09/11/1996 & $<10$ & - & \\
\hline & & & 04/10/1997 & $<10$ & - & \\
\hline & & & $10 / 30 / 2001$ & 4 & - & \\
\hline \multirow[t]{5}{*}{ Utah 11-52 } & 393154 & 1105234 & 09/14/1995 & 220 & - & South of Price \\
\hline & & & 09/11/1996 & $<10$ & - & \\
\hline & & & 04/10/1997 & $<10$ & - & \\
\hline & & & 09/04/1998 & $<10$ & - & \\
\hline & & & 09/16/1999 & 50 & $<10$ & \\
\hline \multirow[t]{2}{*}{ Utah 8-100 } & 393156 & 1104015 & 09/04/1998 & $<10$ & - & South of Price \\
\hline & & & 09/16/1999 & $<10$ & - & \\
\hline \multirow[t]{5}{*}{ Utah 8-97 } & 393218 & 1104913 & 09/14/1995 & $<10$ & - & South of Price \\
\hline & & & 09/11/1996 & $<10$ & - & \\
\hline & & & 04/10/1997 & $<10$ & - & \\
\hline & & & 09/04/1998 & $<10$ & - & \\
\hline & & & 09/16/1999 & $<10$ & - & \\
\hline \multirow[t]{2}{*}{ Utah 7-57 } & 393220 & 1105100 & 09/14/1995 & 90 & - & South of Price \\
\hline & & & 09/4/1998 & $<10$ & - & \\
\hline \multirow[t]{4}{*}{ Utah 9-413 } & 393230 & 1104842 & $09 / 18 / 2000$ & 5,880 & - & South of Price \\
\hline & & & $10 / 30 / 2001$ & 388 & - & \\
\hline & & & $10 / 15 / 2002$ & 174 & - & \\
\hline & & & $09 / 22 / 2003$ & 4 & - & \\
\hline \multirow[t]{2}{*}{ Utah 11-51 } & 393223 & 1105302 & 09/13/1995 & $<10$ & - & South of Price \\
\hline & & & 10/29/2001 & 1 & - & \\
\hline \multirow[t]{4}{*}{ Utah 1-44 } & 393240 & 1105202 & 09/13/1995 & 80 & - & South of Price \\
\hline & & & 04/10/1997 & $<10$ & - & \\
\hline & & & 09/03/1998 & $<10$ & - & \\
\hline & & & 09/16/1999 & $<10$ & - & \\
\hline \multirow[t]{3}{*}{ Utah 4-129 } & 393250 & 1104849 & 09/13/1996 & $<10$ & - & South of Price \\
\hline & & & 04/08/1997 & $<10$ & - & \\
\hline & & & 09/03/1998 & $<10$ & - & \\
\hline
\end{tabular}


Table 3. Soil-gas methane concentration in multiple samples collected adjacent to selected well sites, Carbon and Emery Counties, Utah, 1995-2003-Continued

\begin{tabular}{|c|c|c|c|c|c|c|}
\hline \multirow[b]{2}{*}{ Site name } & \multicolumn{2}{|c|}{ Location } & \multirow[b]{2}{*}{$\begin{array}{l}\text { Sample } \\
\text { date }\end{array}$} & \multicolumn{2}{|c|}{$\begin{array}{c}\text { Methane concentration } \\
\text { (ppmv) }\end{array}$} & \multirow[b]{2}{*}{ Area } \\
\hline & $\begin{array}{l}\text { Latitude } \\
\text { (ddmmss) }\end{array}$ & $\begin{array}{l}\text { Longitude } \\
\text { (dddmmss) }\end{array}$ & & $\begin{array}{c}\text { Sample } \\
\text { collected } \\
\text { within } \\
10 \text { feet } \\
\text { of well }\end{array}$ & $\begin{array}{c}\text { Sample } \\
\text { collected } \\
\text { more than } \\
10 \text { feet } \\
\text { from well }\end{array}$ & \\
\hline Utah 4-129 & & & $09 / 15 / 1999$ & $<10$ & - & \\
\hline \multirow[t]{6}{*}{ Utah 6-39 } & 393322 & 1105104 & 09/13/1995 & 4,850 & - & South of Price \\
\hline & & & $10 / 26 / 1995$ & $<10$ & - & \\
\hline & & & 09/11/1996 & $<10$ & - & \\
\hline & & & 09/03/1998 & $<10$ & - & \\
\hline & & & $10 / 16 / 2002$ & 3 & - & \\
\hline & & & $09 / 22 / 2003$ & 1 & - & \\
\hline \multirow[t]{4}{*}{ Utah 2-46 } & 393317 & 1105225 & 09/14/1995 & 100 & - & South of Price \\
\hline & & & 09/11/1996 & $<10$ & - & \\
\hline & & & 09/03/1998 & $<10$ & - & \\
\hline & & & 09/16/1999 & $<10$ & - & \\
\hline \multirow[t]{2}{*}{ Utah 5-94 } & 393319 & 1104957 & 09/03/1998 & $<10$ & - & South of Price \\
\hline & & & 09/15/1999 & $<10$ & - & \\
\hline \multirow[t]{7}{*}{ Utah 36-3-4 } & 393410 & 1105154 & $10 / 25 / 1995$ & $>100,000$ & 62,400 & South of Price \\
\hline & & & 09/11/1996 & $<10$ & - & \\
\hline & & & 04/10/1997 & $<10$ & - & \\
\hline & & & 09/03/1998 & $<10$ & - & \\
\hline & & & $10 / 29 / 2001$ & 19 & - & \\
\hline & & & $10 / 16 / 2002$ & 11 & - & \\
\hline & & & $09 / 22 / 2003$ & 4 & - & \\
\hline \multirow[t]{4}{*}{ Bawden \#1 } & & & $09 / 20 / 2000$ & $<10$ & - & South of Price \\
\hline & & & $10 / 29 / 2001$ & 3 & - & \\
\hline & & & 09/09/2002 & 2 & - & \\
\hline & & & $09 / 25 / 2003$ & 1 & - & \\
\hline \multirow[t]{3}{*}{ Utah 1} & 393510 & 1104951 & $09 / 20 / 2000$ & $<10$ & - & South of Price \\
\hline & & & $10 / 29 / 2001$ & 1 & - & \\
\hline & & & 09/09/2002 & 2 & - & \\
\hline \multirow[t]{7}{*}{ Utah 19-14-12 } & 393526 & 1105052 & 09/08/1995 & $<10$ & - & South of Price \\
\hline & & & 09/01/1998 & $<10$ & - & \\
\hline & & & 09/15/1999 & $<10$ & - & \\
\hline & & & 09/20/2000 & $<10$ & - & \\
\hline & & & $10 / 29 / 2001$ & 1 & - & \\
\hline & & & 09/09/2002 & 4 & - & \\
\hline & & & $09 / 23 / 2003$ & - & 3 & \\
\hline \multirow[t]{2}{*}{ Utah 19-171 } & 393522 & 1105705 & 04/08/1997 & $<10$ & - & South of Price \\
\hline & & & 09/15/1999 & $<10$ & - & \\
\hline \multirow[t]{2}{*}{ Utah 19-77 } & 393545 & 1105104 & 09/11/1996 & $<10$ & - & South of Price \\
\hline & & & $09 / 20 / 2000$ & $<10$ & - & \\
\hline \multirow[t]{3}{*}{ Utah 22-76 } & 393550 & 1105343 & 09/12/1995 & 660 & - & South of Price \\
\hline & & & 09/11/1996 & $<10$ & - & \\
\hline & & & 09/03/1998 & $<10$ & - & \\
\hline \multirow[t]{4}{*}{ Utah 18-93 } & 393614 & 1105106 & $09 / 20 / 2000$ & 12,400 & - & South of Price \\
\hline & & & $10 / 29 / 2001$ & 6 & - & \\
\hline & & & 09/09/2002 & 465 & - & \\
\hline & & & $09 / 23 / 2003$ & 2 & - & \\
\hline
\end{tabular}


Table 3. Soil-gas methane concentration in multiple samples collected adjacent to selected well sites, Carbon and Emery Counties, Utah, 1995-2003-Continued

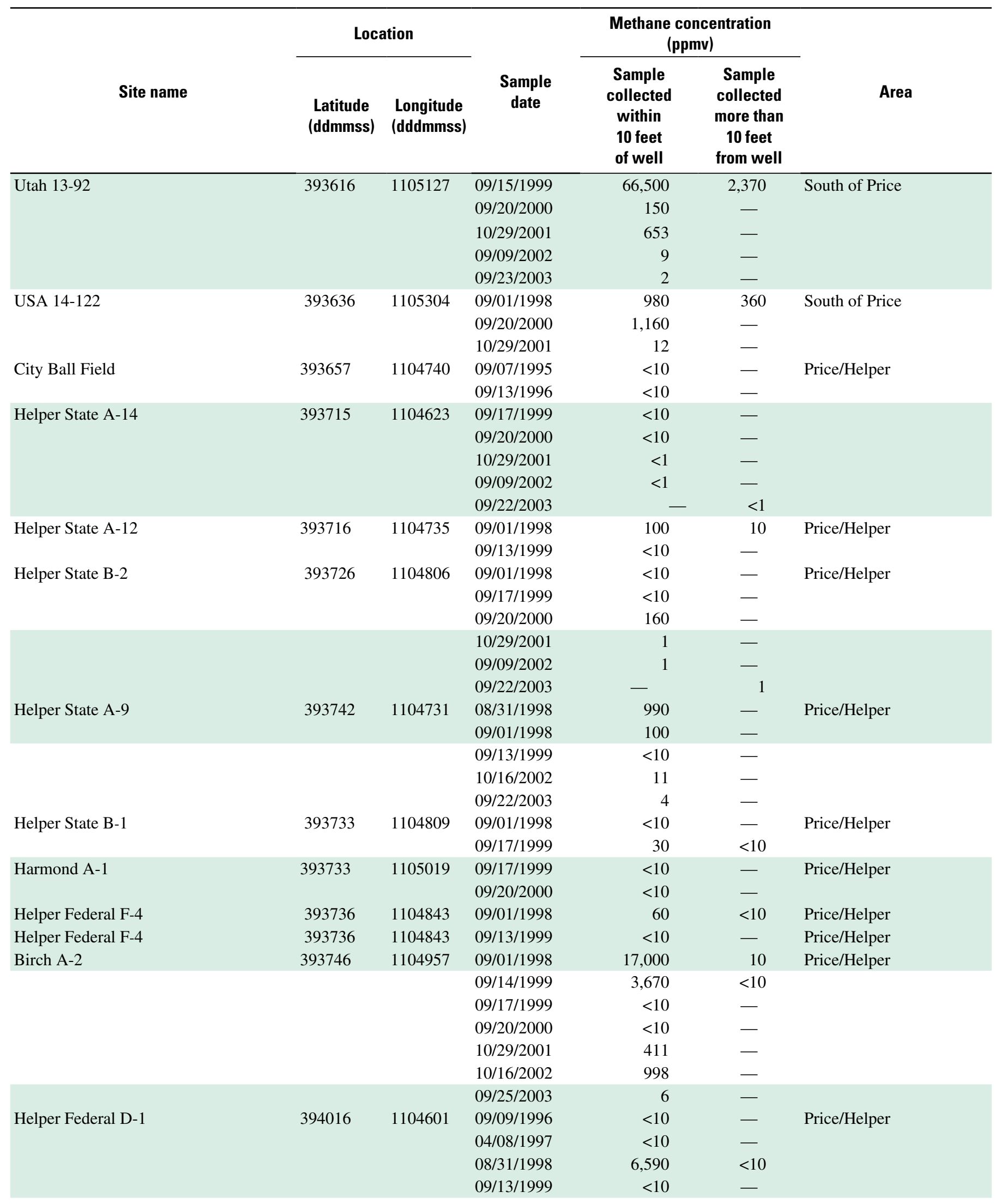


Table 3. Soil-gas methane concentration in multiple samples collected adjacent to selected well sites, Carbon and Emery Counties, Utah, 1995-2003-Continued

\begin{tabular}{|c|c|c|c|c|c|c|}
\hline \multirow[b]{2}{*}{ Site name } & \multicolumn{2}{|c|}{ Location } & \multirow[b]{2}{*}{$\begin{array}{l}\text { Sample } \\
\text { date }\end{array}$} & \multicolumn{2}{|c|}{$\begin{array}{c}\text { Methane concentration } \\
\text { (ppmv) }\end{array}$} & \multirow[b]{2}{*}{ Area } \\
\hline & $\begin{array}{l}\text { Latitude } \\
\text { (ddmmss) }\end{array}$ & $\begin{array}{l}\text { Longitude } \\
\text { (dddmmss) }\end{array}$ & & $\begin{array}{c}\text { Sample } \\
\text { collected } \\
\text { within } \\
10 \text { feet } \\
\text { of well }\end{array}$ & $\begin{array}{c}\text { Sample } \\
\text { collected } \\
\text { more than } \\
10 \text { feet } \\
\text { from well }\end{array}$ & \\
\hline \multirow[t]{9}{*}{ Helper Federal A-1 } & 394044 & 1104632 & $09 / 20 / 1995$ & 38,400 & - & Price/Helper \\
\hline & & & 09/13/1996 & $>100,000$ & - & \\
\hline & & & 04/08/1997 & $<10$ & - & \\
\hline & & & 08/31/1998 & 100 & $<10$ & \\
\hline & & & 09/13/1999 & $<10$ & - & \\
\hline & & & $09 / 20 / 2000$ & $<10$ & - & \\
\hline & & & $10 / 29 / 2001$ & 6 & - & \\
\hline & & & 09/09/2002 & 7 & - & \\
\hline & & & $09 / 25 / 2003$ & - & 9 & \\
\hline \multirow[t]{2}{*}{ Helper Federal A-2 } & 394038 & 1104724 & 08/31/1998 & $<10$ & $<10$ & Price/Helper \\
\hline & & & 09/13/1999 & $<10$ & - & \\
\hline \multirow[t]{2}{*}{ Helper Federal C-1 } & 394058 & 1104655 & 08/31/1998 & 110 & - & Price/Helper \\
\hline & & & 09/13/1999 & $<10$ & $<10$ & \\
\hline
\end{tabular}


Table 4. Soil-gas methane concentration in samples collected at selected ground-water sites, Carbon and Emery Counties, Utah, $1995-2002$

[Site name: Utah Division of Oil, Gas, and Mining designations or field notes. Location: d, degree; m, minute; s, second. Methane concentration: ppmv, parts per million by volume. Samples collected from 1995 through 2000 were analyzed by the OVA 128 Century Organic Vapor Analyzer, which was considered for this monitoring program to have a minimum detection limit of 10 ppmv. Samples collected from 2001 and 2002 were analyzed by the HNU Systems Inc. Model 311 Portable Gas Chromatograph, which was considered for this monitoring program to have a minimum detection limit of 1 ppmv. When no methane was detected at a sample site, a value of less than $10 \mathrm{ppmv}(<10)$ is reported]

\begin{tabular}{|c|c|c|c|c|}
\hline \multirow[b]{2}{*}{ Site name } & \multicolumn{2}{|c|}{ Location } & \multirow[b]{2}{*}{ Sample date } & \multirow{2}{*}{$\begin{array}{c}\text { Methane } \\
\text { concentration } \\
\text { (ppmv) }\end{array}$} \\
\hline & $\begin{array}{l}\text { Latitude } \\
\text { (ddmmss) }\end{array}$ & $\begin{array}{l}\text { Longitude } \\
\text { (dddmmss) }\end{array}$ & & \\
\hline Canyon above Standardville (by sheep pen) spring discharge from cliff face & 394120 & 1105301 & 09/07/1995 & $<10$ \\
\hline Canyon above Standardville Finkbinder Spring & 394116 & 1105405 & 09/07/1995 & $<10$ \\
\hline Covered Old Mine entrance just North of Kenilworth & 394115 & 1105404 & 09/07/1995 & $<10$ \\
\hline Golf Course Spring in the Pond & 393837 & 1105149 & 09/12/1995 & $<10$ \\
\hline Anadarko Office "CPF" & 394747 & 1104609 & 09/18/1995 & $<10$ \\
\hline Spring near Box Spring (NW) in Section 1- Matts Summit Quad & 394823 & 1105227 & 09/19/1995 & $<10$ \\
\hline Shimmin Trust 14-12 (Water sample from lot) & 394732 & 1104511 & 09/19/1995 & $<10$ \\
\hline Clawson Spring - Poison Spring Quad & 392924 & 1105702 & 09/21/1995 & 30 \\
\hline Spring on east side of Kyune Quad Block 35 & 394908 & 1105247 & 09/21/1995 & $<10$ \\
\hline Spring-fed pond by Desert Lake (Elmo Area) & 392202 & 1104330 & $10 / 23 / 1995$ & 130 \\
\hline Irrigation drain & 392332 & 1104625 & $10 / 23 / 1995$ & $<10$ \\
\hline Irrigation drain (shallow ground water) & 392340 & 1104622 & $10 / 23 / 1995$ & $<10$ \\
\hline Spigot at spring/seep source & 391257 & 1104053 & $10 / 23 / 1995$ & $<10$ \\
\hline Staker Spring & 391550 & 1104435 & $10 / 26 / 1995$ & $<10$ \\
\hline \multirow[t]{3}{*}{ Unnamed spring } & 393324 & 1110022 & $09 / 18 / 2000$ & 26,200 \\
\hline & & & $10 / 30 / 2001$ & 560 \\
\hline & & & $10 / 14 / 2002$ & 160 \\
\hline
\end{tabular}




\section{Appendix}

This section describes the collection and analysis procedures used for soil-gas and ground-water samples collected during the 1995-2003 monitoring period. Samples were analyzed for methane using two analytical instruments: the (1) OVA 128 Century Organic Vapor Analyzer and (2) HNU 311 Portable Gas Chromatograph. Sample-collection techniques were the same for both analytical instruments. The desciptions are detailed enough so that any future monitoring can be designed to be comparable to the monitoring program described in this report.

\section{Sample Collection}

Gas samples were extracted from the soil by hand-pounding a perforated steel tube into the ground. The tube has a 3/8-in. outside diameter (OD) and is $4 \mathrm{ft}$ long; perforations are miscellaneous 3/16-in. holes drilled in the bottom $1 \mathrm{ft}$ of the tube. The tip of the steel tube is driven to a depth of 2 to $4 \mathrm{ft}$ below land surface. Once the tube is in the ground, the void (annular) space between the tubing and the soil at land surface is sealed by wiggling the tube until fine-grained material fills in the void space. About 0.75 liter (L) of water is poured around the probe to create the seal. At some locations, the ground is hard and the task of sealing the interface is difficult. If a good seal is not obtained, a notation is recorded on the field form. A bad seal could result in an incorrect measurement of soil gas as a result of contamination of ambient air from above ground getting pulled into the subsurface along the annular space.

In ground water, methane concentration was measured by analyzing the headspace gas in a vial (bottle) of sample water. Two or three vials are gently rinsed, filled with sample water, and sealed with septum caps. The samples are visually inspected to ensure that there are no air bubbles in the bottle. Fifty milliliters (ml) of ambient air are injected through the septum into each bottle with a glass syringe. A second needle (without an attached syringe) also is inserted through the septum to allow displaced water to flow out of the bottle. Once the headspace is created, the needle and syringe are removed, and the vials are set aside for 20 minutes to allow the headspace to equilibrate with the dissolved methane in the water. After that time, a known volume of air is removed from the headspace through the septum with a glass syringe. The withdrawn air is then injected into the analysis equipment.

\section{Analysis Procedures for the OVA 128 Century Organic Vapor Analyzer}

The OVA 128 Century Organic Vapor Analyzer is equipped with a gas chromatograph column to separate methane from other hydrocarbon gases. The OVA 128 uses a hydrogen flame ionization detector (FID). This type of detector is a carbon-molecule counter. Gas samples are injected directly into the OVA 128 with a syringe. The OVA 128 startup procedure is described in the instruction manual. The OVA 128 is a delicate instrument and is transported in the cab of the field vehicle and disconnected from all external gas-supply lines between site visits. The OVA 128 is calibrated once a week during field-data collection and checked daily. Calibration is done by using a 95 parts per million by volume (ppmv) methane-to-air standard. Instrument responses to known methane amounts are compiled in a calibration plot that relates a unitless peak height to the mass of methane in a sample volume of soil gas. The calibration process is discussed in detail by Chafin and others (1993) and summarized in Naftz and others (1998).

Soil gas is collected by means of a small vacuum pump hooked to a short flexible hose that is attached to the top of a perforated steel tube that has been driven into the ground (see "Sample collection" section of the appendix). The pump is run for 2 minutes to purge the tube and hose. A known gas-sample volume is withdrawn from an inline-septum-sample port with a glass syringe and injected into the OVA 128. A unitless peak-height response is recorded. Peak height is converted to concentration with the calibration curve, as explained in Chafin and others (1993) and Naftz and others (1998). For ground water, the dissolved gas is separated from the water sample by using a gas bottle sealed with a septum cap (see "Sample collection" section of appendix). A known gas-sample volume is withdrawn from the sample-bottle headspace through the septum with a glass syringe and injected into the OVA 128. As with soil gas, a unitless peak height is recorded and converted to a methane concentration.

\section{Analysis Procedures for the HNU 311 Portable Gas Chromatograph}

The HNU 311 Portable Gas Chromatograph (GC) used for the monitoring program was equipped with a packed column, an FID, and a 1-cubic-centimeter $\left(\mathrm{cm}^{3}\right)$ sample loop. Control of the HNU 311 is automated and the parameters are set on a computer with Peakworks software. Communication to the HNU 311 is made by using an RS 232 cable connected to PORT 1. Setup communications on the PC are as follows:

MY COMPUTER $\rightarrow$ CONTROL PANEL $\rightarrow$ SYSTEM DEVICE MANAGER $\rightarrow$ PORTS $\rightarrow$ COM $1 \rightarrow$ PORT SETTINGS $\rightarrow$ bits per second $=9,600$

Once the communications port has been set up, the gas chromatograph parameters must be adjusted. From the menu bar, select Method. From the Method drop-down menu, select the Edit dialog box and set the following parameters:

\begin{tabular}{cc}
\hline Parameter & Setting \\
\hline INJ/DET Temp & 110 \\
Oven Temp & 50 \\
Analysis Time & $1: 30$
\end{tabular}




\author{
${ }^{1}$ Sample Time 2:00 \\ Inject Time \\ $0: 15$
}

For the remaining parameters, use the default setting. After exiting the dialog box, save the job file using the File drop down menu. Every time a dialog box is exited, save the job file.

From the menu bar, select Method. From the Method drop-down menu, select the Detector A dialog box, turn on the detector, and set the following parameters:

\begin{tabular}{cc}
\hline Parameter & Setting \\
\hline Length & $0: 30$ \\
Segment Width & 10 \\
Units & PPM \\
Range & 100 \\
\hline
\end{tabular}

For the remaining parameters, use the default setting.

Continuing from the Method drop-down menu, select the

Detector B dialog box and turn off the detector.

From the menu bar, select Method. From the Method drop-down menu, select the Components table, select the Edit dialog box, toggle on, and set the following parameters:

\begin{tabular}{cc}
\hline Parameter & Setting \\
\hline Name & Methane \\
Peak RT & $0: 39$ \\
Window & $0: 05$ \\
\hline
\end{tabular}

For the remaining parameters, use the default setting.

Continuing from the Method drop-down menu, select the Standards table and highlight the Methane record. Select the Edit dialog box and set the following parameter:

\begin{tabular}{cc}
\hline Parameter & Setting \\
\hline Stand. 0 & ${ }^{2} 9.78$ \\
\hline
\end{tabular}

Now, from the Options drop-down menu, select the Preferences dialog box and set the following parameter:

\begin{tabular}{cc}
\hline Parameter & Setting \\
\hline Port & COM 1: \\
\hline
\end{tabular}

Remember to save the job file using the File drop down menu.

If the HNU 311 starts to beep and the beeping cannot be stopped, the power must be manually cycled. This is a known HNU 311 bug. Follow the steps below:

Remove the HNU 311 from case.

Disconnect ground cable.

On left side top, slide out first circuit board and push it back in. This power cycles the board and should stop the beeping.

${ }^{1}$ This is the time that the HNU 311 pumps in a sample, maximum flow rate is $250-300$ cubic centimeters per minute $\left(\mathrm{cm}^{3} / \mathrm{min}\right)$ (p. 3-9 in the HNU 311 instruction manual). Sample time needs to be long enough to ensure that three sample volumes have been pumped through the sample rod and connecting plumbing.

${ }^{2}$ This value needs to match the methane concentration of the gas mixture used for calibration of the gas chromatograph.
In the field, the HNU 311 is calibrated each day prior to any sample collection and intermittently throughout the day depending on drift, response, and circumstances. If the standard is different from that used during initial setup, the concentration is adjusted with Peakworks. The HNU 311 is a delicate instrument and is transported in the cab of the field vehicle and disconnected from all external gas-supply lines between site visits. The oven is turned off between sites, but a small amount of nitrogen (from the internal tank) is kept flowing through the column to cool it down and prevent ambient air from diffusing into the column. The HNU 311 is powered by a gas generator with a rating of at least 2,000 watts. Ultra-pure-grade nitrogen is the carrier gas, and hydrogen gas is used for the FID. Standard-size cylinders containing each of these gases are transported to the field to refill the smaller internal HNU 311 gas bottles (as described in the instruction manual for HNU 311).

Calibration gas enters the instrument at the inlet port labeled "CAL GAS IN" through a short tube hooked directly to the calibration gas pressure tank. Soil-gas samples are inlet to the HNU 311 at the port labeled "SAMPLE PUMP IN" by using a 30 -ft length of $1 / 8$-in. OD gas-impermeable nylon tubing attached to the top of a perforated steel tube that has been driven into the ground (see "Sample collection" section of the appendix). The HNU 311 has an internal pump that pulls a sample in through the tubing. A small air filter (or piece of foam) is inserted in the line between the steel tube and the 1/8-in. nylon tubing to prevent dust and other detritus from being sucked into the HNU 311. Flow rate of the internal pump is $250-300 \mathrm{~cm}^{3} /$ minute at the inlet (p. 3-9 of the instruction manual) and the tube is pumped for 2 minutes to ensure that air in the tube is from the surrounding soil and that there is no residual methane in the steel tube and line from previous samples or ambient air. The gas volumes in the steel and connecting tubing are:

Volume is calculated as $\mathrm{Pi}^{*}$ radius ${ }^{2 *}$ length. Based on outside diameter, the steel tubing volume is:

$$
\begin{aligned}
& (0.375 \mathrm{in} * 2.54 \mathrm{~cm} / \mathrm{in} * 0.5)^{2} * 3.142=0.713 \mathrm{~cm}^{2} * 4 \\
& \mathrm{ft} * 30.48 \mathrm{~cm} / \mathrm{ft}=87 \mathrm{~cm}^{3} .
\end{aligned}
$$

Based on outside diameter, the connecting tubing volume is:

$$
(0.125 \mathrm{in} * 2.54 \mathrm{~cm} / \mathrm{in} * 0.5)^{2} * 3.142=0.079 \mathrm{~cm}^{2} * 30
$$$$
\mathrm{ft} * 30.48 \mathrm{~cm} / \mathrm{ft}=72 \mathrm{~cm}^{3} \text {. }
$$

Consecutive readings are made at a site until there is a discrepancy of less than 10 percent between readings. Normally, this requires three to six measurements. The last measurement is reported as the methane soil-gas concentration for the site. Local ambient-air methane measurements were obtained after the analysis of soil gas.

For ground water, the dissolved gas is separated from the water sample by using a gas bottle capped with septum (see "Sample collection" section of the appendix). A known gassample volume is withdrawn from the sample-bottle headspace 
through the septum with a glass syringe and injected into the HNU 311 at the injection port (fig. 3.1 of the instruction manual). When samples are manually injected rather than pumped (as is the situation for soil-gas samples), operation of the HNU 311 must be modified with the Peakworks software. From the menu bar, select Method. From the Method drop-down menu, select the Edit dialog box and toggle on Syringe Inj. 
Methane Gas Concentration in Soils and Ground Water, Carbon and Emery Counties, Utah, 1995-2003 


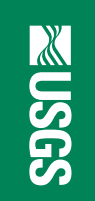

옴

를

옿

कs

京

()

क

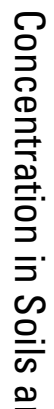

ํㅡㄹ

몬

응

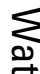

ᄋ

음

을

罡

$\varnothing$

离.

$\stackrel{Ð}{Ð}$

cै

సે요

ए

刃

옹

స్త 